ORIGINAL ARTICLE

\title{
A combination of physical activity and computerized brain training improves verbal memory and increases cerebral glucose metabolism in the elderly
}

\author{
T Shah $^{1,2,3}$, G Verdile ${ }^{2,3,4}$, H Sohrabi ${ }^{1,2,3}$, A Campbell ${ }^{5}$, E Putland ${ }^{2}$, C Cheetham $^{6,7}$, S Dhaliwal $^{8}$, M Weinborn $^{2,9}$, P Maruff $^{10,11}$, \\ D Darby ${ }^{10,11,12}$ and RN Martins ${ }^{1,2,3}$
}

\begin{abstract}
Physical exercise interventions and cognitive training programs have individually been reported to improve cognition in the healthy elderly population; however, the clinical significance of using a combined approach is currently lacking. This study evaluated whether physical activity (PA), computerized cognitive training and/or a combination of both could improve cognition. In this nonrandomized study, 224 healthy community-dwelling older adults (60-85 years) were assigned to 16 weeks home-based PA $(n=64)$, computerized cognitive stimulation $(n=62)$, a combination of both (combined, $n=51)$ or a control group ( $n=47)$. Cognition was assessed using the Rey Auditory Verbal Learning Test, Controlled Oral Word Association Test and the CogState computerized battery at baseline, 8 and 16 weeks post intervention. Physical fitness assessments were performed at all time points. A subset (total $n=45$ ) of participants underwent $\left[{ }^{18} \mathrm{~F}\right]$ fluorodeoxyglucose positron emission tomography scans at 16 weeks (postintervention). One hundred and ninety-one participants completed the study and the data of 172 participants were included in the final analysis. Compared with the control group, the combined group showed improved verbal episodic memory and significantly higher brain glucose metabolism in the left sensorimotor cortex after controlling for age, sex, premorbid IQ, apolipoprotein E (APOE) status and history of head injury. The higher cerebral glucose metabolism in this brain region was positively associated with improved verbal memory seen in the combined group only. Our study provides evidence that a specific combination of physical and mental exercises for 16 weeks can improve cognition and increase cerebral glucose metabolism in cognitively intact healthy older adults.
\end{abstract}

Translational Psychiatry (2014) 4, e487; doi:10.1038/tp.2014.122; published online 2 December 2014

\section{INTRODUCTION}

The rise in life expectancy has led to increases in the prevalence of dementia globally. ${ }^{1}$ Lifestyle plays a vital role in increasing the risk of dementia, as poor diet and inactivity-both physical and mental-have been associated with cognitive decline. ${ }^{2-4}$ It has been estimated that the prevalence of dementia will increase over fourfold by $2050,{ }^{5}$ and so far, pharmacological interventions have been ineffective. As a result, lifestyle interventions have attracted substantial interest, as physical and mental inactivity including poor diet are risk factors that are modifiable. Specifically, studies indicate that increasing the mental and physical activity, improving diet and changing an individual's response to stressful stimuli are avenues through which we can maintain brain health and lower the risk of dementia. ${ }^{6-8}$

Observational studies have reported that physical activity $(P A)$ is associated with better cognitive performance and reduced dementia risk. ${ }^{9-12}$ Intervention studies and meta-analysis of PA clinical trials for healthy older adults report enhanced memory, executive function and reaction time, ${ }^{13-15}$ including improved cognition in Alzheimer's disease (AD) patients. ${ }^{16,17}$ A study $(n=170)$ reported that 6 months of walking intervention improved ADAS-Cog scores in patients with mild cognitive impairment $(\mathrm{MCl})$ compared with the control group. ${ }^{18}$ Aerobic exercise intervention is most beneficial for cognition, ${ }^{19}$ whereas resistance training has shown mixed results. ${ }^{20,21}$ Like PA, interventions using mentally stimulating/training (for example, reality orientation, method of loci, mental imagery and so on) activities also enhance cognition in healthy older adults, ${ }^{22-24}$ people with $\mathrm{MCl}^{25}$ and in $\mathrm{AD}$ patients, ${ }^{26-28}$ and is associated with reduced dementia risk. ${ }^{29,30}$ The Advanced Cognitive Training for Independent and Vital Elderly and the Improvement in Memory with Plasticity-based Adaptive Cognitive Training clinical trials have provided the best evidence of benefits from computerized brain training for verbal memory, reasoning, processing speed and attention. ${ }^{22,31,32}$ Recently, a study which used video-game format (NeuroRacer) as cognitive training intervention showed that older adults gained

\footnotetext{
${ }^{1}$ School of Psychiatry and Clinical Neurosciences, University of Western Australia, Perth, WA, Australia; ${ }^{2}$ The McCusker Alzheimer's Research Foundation (Hollywood Private Hospital), Hollywood Medical Centre, Perth, WA, Australia; ${ }^{3}$ Centre of Excellence for Alzheimer's Disease Research and Care, School of Medical Sciences, Edith Cowan University, Perth, WA, Australia; ${ }^{4}$ School of Biomedical Sciences, Curtin University, Perth, WA, Australia; ${ }^{5}$ Department of Nuclear Medicine, Royal Perth Hospital, Perth, WA, Australia; ${ }^{6}$ Health Care Western Australia, Hollywood Private Hospital, Perth, WA, Australia; ${ }^{7}$ School of Sports Science, Exercise and Health, University of Western Australia, Perth, WA, Australia; ${ }^{8}$ School of Public Health, Curtin University, Perth, WA, Australia; ${ }^{9}$ School of Psychology, University of Western Australia, Perth, WA, Australia; ${ }^{10}$ Mental Health Research Institute, The University of Melbourne, Parkville, VIC, Australia; ${ }^{11}$ CogState Ltd, Melbourne, VIC, Australia and ${ }^{12}$ Florey Neuroscience Institutes, Carlton South, VIC, Australia. Correspondence: Professor RN Martins, Centre of Excellence for Alzheimer's Disease Research and Care, School of Medical Sciences, Edith Cowan University, 270 Joondalup Drive, Joondalup, Perth, WA 6027, Australia.
}

E-mail: r.martins@ecu.edu.au

The findings of this study were orally reported at the Society for Neuroscience (SFN) Conference, 2013; San Diego, CA, USA

Received 28 April 2014; revised 19 September 2014; accepted 5 October 2014 


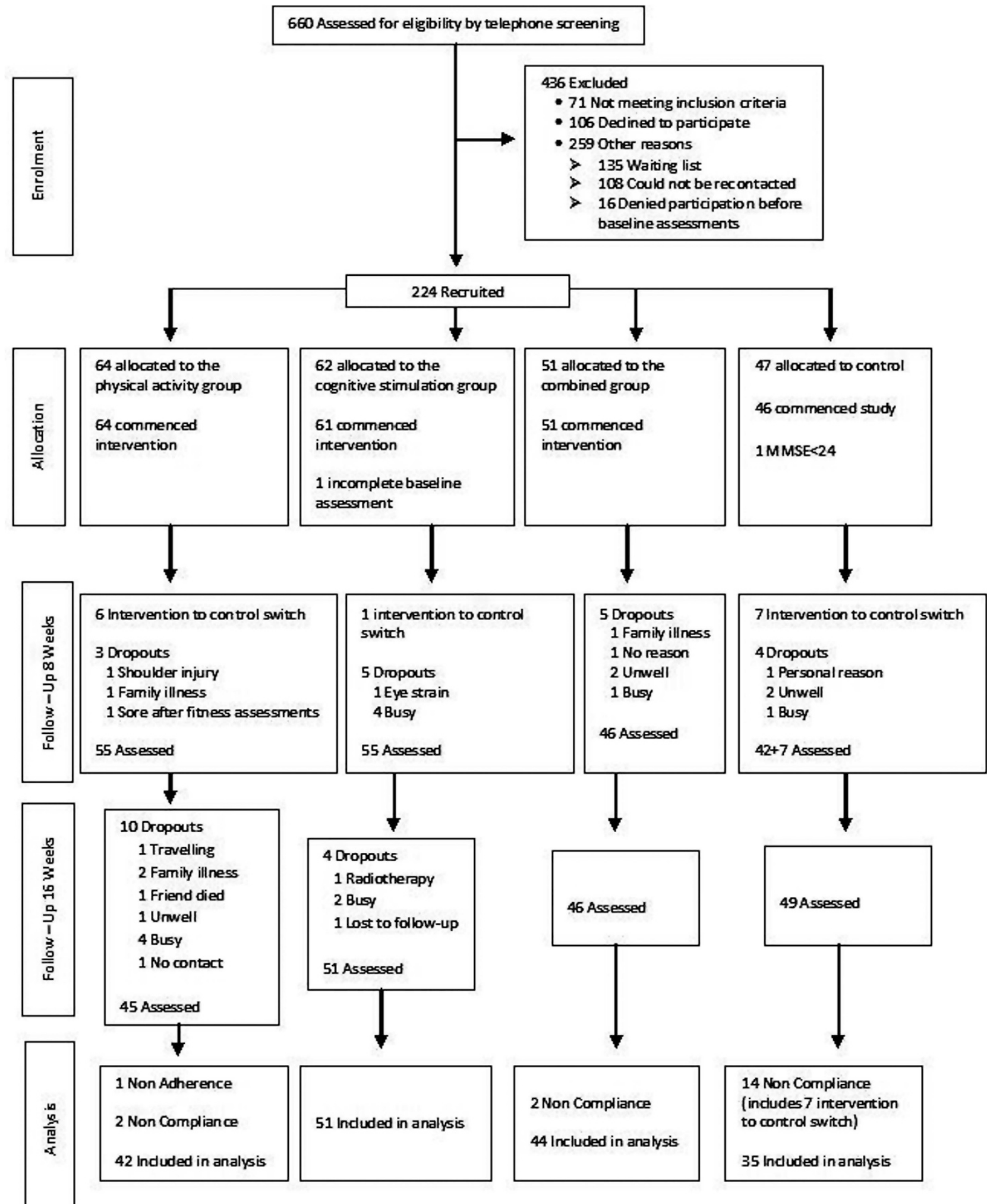

Figure 1. Flow of participants from screening to completion of the 16 weeks post-intervention follow-up assessment.

enhanced cognitive control along with improved untrained cognitive control abilities. The study demonstrated that agerelated deficits in neural control of cognition were associated with enhanced midline frontal theta power and frontal-posterior theta coherence using electroencephalography; thus highlighting the plasticity of this region in the aging brain. ${ }^{33}$

Although the benefits of physical or mental activities on cognition have empirical support, whether further improvement is achieved by carrying out both activities has not been widely 
explored. Limited clinical studies ${ }^{34-37}$ assessing combined effects have used only cognitive parameters with no inclusion of biomarkers related to AD. Cerebral glucose metabolism, as measured by $\left[{ }^{18} \mathrm{~F}\right]$ fluorodeoxyglucose (FDG) positron emission tomography (PET), is tightly connected to neuronal activity. It can be used as a marker of synaptic dysfunction before neurodegeneration advances further ${ }^{38,39}$ and in fact, cerebral glucose hypometabolism has been reported in $\mathrm{MCl}^{40,41}$ and $A D$ patients. $^{42-45}$

A recent study reported that 6 months of cognitive training increased cerebral glucose metabolism in $\mathrm{MCl}$ patients only; however, there were no significant associations between increased FDG uptake and improved cognition. ${ }^{46}$ PA has also been shown to alter brain glucose metabolism; ${ }^{47,48}$ however, these studies appear to be limited to conditions of high intensity exercise in young middle-aged cognitively normal cohorts. Studies that include neuroimaging assessments of combined interventions in healthy elderly subjects are currently lacking.

The current study tested the hypothesis that superior cognitive benefits occur when using a combination of physical and mental training. The method involved studies of cognitively intact older adults, divided into four groups: PA, cognitive stimulation (CS), combined and a control group. Besides assessing cognition, FDG-PET was used for assessing neuronal activity at week 16 post intervention.

\section{MATERIALS AND METHODS}

\section{Participants}

The study was a single site-controlled, nonrandomized trial conducted between June 2009 and December 2011. Participants were recruited from either (1) an existing database of healthy individuals at the McCusker Alzheimer's Research Foundation, Hollywood Private Hospital (HPH), Perth, WA, Australia, (2) the Survey Research Centre, Edith Cowan University (ECU; June 2010) or (3) through local media advertisements, public talks and word of mouth. The study was approved by the Human Research Ethics Committees of ECU, HPH and the University of Western Australia.

\section{Eligibility, inclusion and exclusion criteria}

Males and females, aged 60-85 years old and providing informed consent, were recruited. Exclusion criteria included inability to communicate in English, visual or auditory impairment, MMSE (Mini Mental State Examination) $\leqslant 24$, diagnosis of dementia, uncontrolled medical conditions that could lead to difficulty complying with the protocol including history of cardiovascular disorders, epilepsy and arthritis causing disability. Participants were required to provide a physician's certificate to allow exercise participation, and were required to have access to, and be comfortable using a personal computer with internet connection.

\section{Study interventions}

Six hundred and sixty participants were screened for eligibility over the telephone. Figure 1 shows the flow of the participants from screening through to the post-intervention follow-up at week 16. Two hundred and twenty-four participants consented to take part in the study. One participant had an MMSE $<24$ and one participant did not complete baseline assessments. Hence, 222 participants commenced the study. Participants were recruited into four groups; PA, CS, both PA and CS (combined) and control. Monthly group meetings were conducted, log books given and fortnightly phone calls made during the 16-week intervention. Group discussions ensured fulfillment of study protocol and resolution of any technical problems.

Though initial attempts were made to randomize subjects to treatment groups, the preferences of some of the subjects made the objective difficult as randomizing them put at risk the viability of the study due to the increased possibility of noncompliance and nonadherence. Thus, participants were allocated to the intervention and control arms alternatively with $<10 \%$ participants being allocated into groups on the basis of their preference (reasons being couples, friends, relatives, busy schedule and so on). The baseline data of all treatment groups were compared to ensure that no biases were introduced as a result of self- selection. In addition, baseline value of the primary outcome variables were included as covariate in the respective analysis.

Instructions on and training for intervention-specific activities were provided by an instructor in the monthly intervention-specific group meeting. All physical activities were performed under the training provided by a team of exercise physiologists in the group meetings. Instructions on performing respective cognitive exercises were also given for those in the CS group. Instruction booklets with detailed explanation of respective physical and cognitive exercises were given. The different home-based activities carried out by the groups are outlined below:

PA group. PA consisted of 48 sessions of walking for $60 \mathrm{~min}$ per day, 3 days per week and 32 sessions of resistance training for $40 \mathrm{~min}$ per day, 2 days per week. Exercises included 10 min sessions for warming up and cooling down. Resistance training incorporated all major muscle groups using an elastic tube of $25 \mathrm{~kg}$ of resistance known as the Gymstick (www. gymstick.net) for upper (for example, chest press, seated row, biceps curl) and ankle weights for lower (for example, leg press, leg extension, leg curls) body exercises as tolerated. Each participant was instructed to record their rate of perceived exertion (Borg Scale ${ }^{49}$ ) during the activities. The intensity of the exercises were gradually increased under the supervision of an exercise physiologist at each group meeting.

CS group. CS consisted of 40 sessions each of the auditory-based Brain Fitness Program (BFP) and the visual-based Insight Program (IP) (Posit Science; San Francisco, CA, USA) for 60 min per day, 5 days a week. Half the participants commenced with BFP for the first 8 weeks and IP for the next 8 weeks and vice versa for the remaining half of the participants.

$P A+C S$ group. The combined activity consisted of both PA and CS sessions (as described above) each carried out for $60 \mathrm{~min}$ per day, 5 days per week, totaling 160 combined sessions.

Control group. This group continued with their routine lifestyle activities. Logbooks were provided to record their physical and mental recreational activities for 16 weeks.

\section{Compliance and adherence}

Compliance was monitored via the daily logbook and by fortnightly phone calls, and adherence rate was calculated on the basis of the number of training sessions completed and recorded in the logbook. Nonadherence was defined as any participant that completed $<25 \%$ of the total number of respective group sessions at the end of the 16-week intervention. Noncompliance was considered as any participant changing their behavior despite the request to maintain their usual lifestyle pattern or the allocated intervention exercises. This included those participants allocated to the control group commencing activities as a result of the information obtained from the study and the fact that there were three other intervention groups, and the intervention participants performing more robust activities outside their routine lifestyle and the allocated daily activities.

\section{Adverse events}

Eight participants reported adverse events that were considered as possibly study-related (shoulder injury due to falling over the carpet while doing fitness assessment in one, sore arms and legs in five, ringing sensation in head with eye strain in one and sweating and exhaustion in one). All participants recovered except the participant with shoulder injury who underwent treatment.

\section{Assessments}

Neuropsychological and physical fitness assessments were administered at baseline, 8 and 16 weeks of the study. Blood samples were collected to harvest DNA from leukocytes for APOE genotyping. ${ }^{50}$ The primary outcome measure of the study was cognition. Secondary outcome measures included changes in self-reported questionnaires, fitness assessments and differences in the cerebral glucose metabolism in the intervention groups versus the control group. The research staff involved in the enrollment and outcome assessment were unblinded to group assignments as they assisted in organizing the monthly interventionspecific group meetings and follow-up phone calls. 
Assessment of cognitive function, anxiety, depression and quality of life

Assessments included the Cambridge Contextual Reading Test ${ }^{51}$ for Premorbid IQ, the Rey Auditory Verbal Learning Test ${ }^{52}$ for verbal episodic memory; Controlled Oral Word Association Test ${ }^{53}$ for verbal fluency and the computerized CogState battery (CogState, Melbourne, VIC, Australia). CogState tasks included Detection (DET) for processing speed, one back memory (ONB) for attention, Groton maze learning for executive functions and Groton maze learning test recall, identification, one card learning and the continuous paired associate learning for assessing the visual memory. As the continuous paired associate learning, identification, Groton maze learning test recall and one card learning tasks assessed visual memory, an index score was calculated using sample-based $z$-scores. Dementia was diagnosed using the Cambridge Cognitive Assessment-Revised ${ }^{54}$ with a cutoff score of $<80 / 105$. The MMSE is a part of Cambridge Cognitive Assessment-Revised. The Hospital Anxiety and Depression Scale ${ }^{55}$ assessed anxiety and depression. Self-report measure of everyday memory functioning was assessed using the Memory Functioning Questionnaire ${ }^{56}$ (MFQ). Healthrelated quality of life was assessed using the physical and mental health component of the SF-36 (short form-36) ${ }^{57}$ questionnaire.

\section{PA and fitness assessments}

Baseline PA levels were assessed using the International Physical Activity Questionnaire. The incremental shuttle walk test ${ }^{58}$ and an assessment of muscular strength using one repetition maximum protocol across four major muscle groups were performed at baseline, 8 and 16 weeks post intervention by the same operator. Exercise intensity levels were determined using the Borg scale. ${ }^{49}$

\section{PET imaging and processing}

Participants $(n=13)$ underwent baseline scans, which were used for postintervention comparison. At week 16 (post intervention), a different subset of participants was scanned from each group. Scans were performed 45 min after intravenous injection of $185 \mathrm{MBq}\left[{ }^{18} \mathrm{~F}\right] \mathrm{FDG}$ on a Philips Gemini 16 Power PET/CT scanner. All participants were randomly selected for PET imaging. The procedure was performed in a quiet, dimly lit room. Images were processed using the NeuroStat brain analysis software package, as described previously. ${ }^{42,44,59,60}$

\section{Data analysis}

Univariate analysis of variance was performed for all continuous variables and chi-squared tests for all categorical variables to compare the four treatment groups. For study outcome variables, the general linear model repeated measures analysis of covariance procedure was used to assess the main effects of the intervention including its interaction with time, after adjusting for the covariates age, sex, APOE status, Cambridge Contextual Reading Test and the respective baseline value. Post hoc comparisons were only performed when group or group $\times$ time interaction was significant. Comparisons between groups were assessed at the $5 \%$ level of significance. Statistical analysis was performed using The Statistical Package for Social Sciences (IBM SPSS version 19). The highly conservative Bonferroni corrections were not used (see Perneger ${ }^{61}$ ). A minimum of 35 subjects in each of the four groups were determined to have $80 \%$ power at $5 \%$ level of difference to detect changes of at least $20 \%$ in primary outcome variables. Additional recruitments were made to replace/foresee that these numbers did not decrease due to dropouts in the study.

\section{PET data analysis}

The PET images were analyzed using NeuroStat brain analysis software as described previously. ${ }^{42,60,62}$ Briefly, each study was transformed using linear scaling and nonlinear warping to match the NeuroStat standard Talairach anatomical atlas, and maximum cortical activity was extracted using the three-dimensional stereotactic surface projection method described by Minoshima et al. ${ }^{42}$ Images were normalized to the mean cortical activity (1000, arbitrary units). To examine significant changes in cerebral glucose metabolism post intervention, week 16 scans on participants (total $n=45$ ) in the three-dimensional stereotactic surface projection images for the four groups were compared separately with the baseline FDG-PET scans $(n=13)$ using a Student's $t$-test on a voxel-byvoxel basis.

$T$-values were converted to equivalent $z$-scores and a statistically significant threshold was used, controlling for multiple pixel comparisons and the shape of the stochastic process on the three-dimensional stereotactic surface projection format, of $z=4.53(P<0.05)$.

NeuroStat was also used to calculate the volume of interest by automatically determining the normalized average surface projection count for major cortical regions (including the parietal, temporal, frontal, occipital, posterior and anterior cingulate cortices) on the week 16 postintervention scans. An analysis of covariance was performed on this data with age, sex, APOE status, Cambridge Contextual Reading Test and history of head injury as covariates. Finally, to determine associations, Spearman's rank correlation was performed with post-intervention neuropsychological scores and regions showing higher cerebral glucose metabolism.

\section{RESULTS}

\section{Descriptive statistics}

Table 1 shows participant $(n=222)$ demographic and clinical characteristics. Overall, the study participants had an average age of $67.60 \pm 5.42$ years and $69 \%$ were females. Baseline group differences after recruitment were observed for the $A P O E \varepsilon 4$ status, Borg's scale and SF-36 physical component with no group differences for other parameters. APOE status was included as a covariate in all analyses, whereas inclusion of Borg's scale and SF-36 physical component as covariates did not alter the main results and hence were removed from the analyses. Seven participants switched from the intervention group to the control group. These participants were considered noncompliant together with 11 other noncompliant participants and were excluded from the final analysis. One participant had $<25 \%$ adherence and 31 participants were dropouts. Thus, data of 172 participants were included and analyzed. After correcting for multiple comparisons with respect to age, sex, APOE and Cambridge Contextual Reading Test including all primary outcome variables, completers $(n=172)$ were not significantly different $(P>0.002)$ than non-completers $(n=52)$.

Effect of the interventions on cognition: a combination of PA and CS improves verbal memory

Cognitive and fitness assessment results following repeated measures analysis of covariance for the four groups are shown in Supplementary Table 1. Significant group differences were observed for the long-term delayed recall (LTDR) using the Rey Auditory Verbal Learning Test $(P=0.040$, partial eta squared 0.055$)$. Post hoc analysis revealed that the combined group performed significantly better $(P=0.024)$ compared with the control group (Figure 2). Group differences were observed for the short-term delayed recall $(P=0.026)$ but when compared with the control group at 8 and 16 weeks; there were no significant changes in the PA $(P=0.560), C S(P=0.809)$ and combined groups $(P=0.966)$ at 8 weeks or at 16 weeks PA $(P=0.328), C S(P=0.724)$ and combined groups $(P=0.309)$, respectively. No significant group or group $x$ time interaction was observed for immediate recall, executive function, processing speed, visual memory or attention.

Significant group differences were observed for the total score of MFQ $(P=0.021)$. Post hoc analysis revealed that the control group reported better memory functioning than the PA $(P=0.027)$ and the combined group $(P=0.016)$. A significant group $\times$ time interaction $(P=0.019)$ was observed for the distance traveled, as assessed by the Shuttle walk test. Compared with the control group at 8 weeks, the combined group $(P=0.020)$ and at 16 weeks the PA group $(P=0.012)$ performed better. No significant changes were observed for measures of SF-36 questionnaire, anxiety and depression scales as well as for the one repetition maximum test and the Borg's scale.

\section{NeuroStat results and analysis of variance}

$P A$ and computerized brain training together increase cerebral glucose metabolism. A total of 45 participants across all groups 
Table 1. Baseline demographics, clinical characteristics and assessments for entire cohort and the four intervention groups ${ }^{\mathrm{a}}$

\begin{tabular}{|c|c|c|c|c|c|c|}
\hline \multirow[t]{2}{*}{ Variables } & \multirow[t]{2}{*}{ Total cohort $(n=222)$} & \multicolumn{4}{|c|}{ Intervention groups } & \multirow[t]{2}{*}{ P-value $e^{b}$} \\
\hline & & $\begin{array}{l}\text { Physical activity, } \\
\qquad \mathrm{n}=42\end{array}$ & $\begin{array}{c}\text { Cognitive stimulation, } \\
\qquad \mathrm{n}=51\end{array}$ & Combined, $\mathrm{n}=44$ & Control, $\mathrm{n}=35$ & \\
\hline Age & $67.60 \pm 5.42$ & $67.36 \pm 5.09$ & $66.61 \pm 4.82$ & $67.18 \pm 5.33$ & $69.06 \pm 5.59$ & 0.187 \\
\hline Females, $n(\%)$ & 153 (68.92) & $29(69.00)$ & $32(62.70)$ & $29(65.90)$ & $26(74.30)$ & 0.716 \\
\hline Education (PG), $n$ (\%) & 35 (15.98) & $5(11.90)$ & $11(22.00)$ & $6(13.60)$ & $6(17.10)$ & 0.094 \\
\hline Retired, $n(\%)$ & 167 (75.57) & $34(81.00)$ & $35(68.60)$ & $32(72.70)$ & $29(82.90)$ & 0.531 \\
\hline High blood pressure, $n(\%)$ & 75 (34.09) & $11(26.20)$ & $16(31.40)$ & $14(31.80)$ & $16(45.70)$ & 0.318 \\
\hline Heart attack, $n(\%)$ & $8(03.62)$ & $0(00.00)$ & $0(00.00)$ & $2(04.50)$ & $3(08.60)$ & 0.066 \\
\hline Diabetes, $n(\%)$ & 26 (11.76) & $6(14.30)$ & $5(09.80)$ & $4(09.10)$ & $4(11.40)$ & 0.589 \\
\hline Visual defects, $n$ (\%) & $132(59.73)$ & $20(47.60)$ & $34(66.70)$ & $28(63.60)$ & $19(54.30)$ & 0.242 \\
\hline History of falls, $n$ (\%) & $15(06.79)$ & $4(09.50)$ & $5(09.80)$ & $3(06.80)$ & $2(05.70)$ & 0.879 \\
\hline Thyroid, $n$ (\%) & $34(15.38)$ & $10(23.80)$ & $3(05.90)$ & $7(15.90)$ & $6(17.10)$ & 0.112 \\
\hline Arthritis, $n$ (\%) & $91(41.18)$ & $14(33.30)$ & $22(43.10)$ & $18(40.90)$ & $11(31.40)$ & 0.624 \\
\hline Joint replacement, $n$ (\%) & $20(09.05)$ & $2(04.80)$ & $5(09.80)$ & $4(09.10)$ & $1(02.90)$ & 0.539 \\
\hline Head injury, $n$ (\%) & $10(04.52)$ & $1(02.40)$ & $4(07.80)$ & $1(02.30)$ & $1(02.90)$ & 0.447 \\
\hline Depression, $n$ (\%) & 37 (16.82) & $7(17.10)$ & $10(19.60)$ & $3(06.80)$ & $5(14.30)$ & 0.339 \\
\hline Anxiety, $n$ (\%) & $31(14.09)$ & $5(12.20)$ & $5(09.80)$ & $6(13.60)$ & $6(17.10)$ & 0.792 \\
\hline Smoking, $n$ (\%) & $12(05.45)$ & $1(02.40)$ & $3(05.90)$ & $3(06.80)$ & $2(05.90)$ & 0.806 \\
\hline Premorbid IQ (CCRT) & $38.13 \pm 6.46$ & $37.29 \pm 6.80$ & $38.39 \pm 6.33$ & $40.25 \pm 4.40$ & $38.26 \pm 6.53$ & 0.149 \\
\hline BMI $(n=212)$ & $27.28 \pm 4.81$ & $26.60 \pm 4.42$ & $28.08 \pm 4.57$ & $27.00 \pm 5.10$ & $26.51 \pm 4.16$ & 0.373 \\
\hline IPAQ $(n=216)$ & $4431.63 \pm 4536.96$ & $4432.11 \pm 5064.37$ & $4294.36 \pm 4042.81$ & $4749.62 \pm 5326.22$ & $3673.00 \pm 3157.38$ & 0.773 \\
\hline APOE $\varepsilon 4$ carriers, $n(\%)$ & $65(29.41)$ & $14(33.30)$ & $18(35.30)$ & $17(38.60)$ & $3(08.60)$ & $0.018^{c}$ \\
\hline \multicolumn{7}{|l|}{ Physical fitness assessments } \\
\hline $\begin{array}{l}\text { Total sum of strength } \\
(\mathrm{kg})(n=211)\end{array}$ & $191.39 \pm 58.18$ & $193.81 \pm 53.51$ & $205.11 \pm 63.14$ & $204.43 \pm 57.01$ & $168.24 \pm 54.03$ & 0.062 \\
\hline Distance $(n=213)$ & $470.52 \pm 138.01$ & $501.19 \pm 129.28$ & $466.38 \pm 114.62$ & $485.68 \pm 136.29$ & $481.76 \pm 143.58$ & 0.659 \\
\hline Borg's Scale $(n=212)$ & $12.02 \pm 2.24$ & $12.32 \pm 2.24$ & $11.55 \pm 2.07$ & $12.50 \pm 2.29$ & $11.03 \pm 2.04$ & $0.011^{c}$ \\
\hline \multicolumn{7}{|l|}{ Assessment score } \\
\hline CAMCOG-R & $96.17 \pm 4.20$ & $96.69 \pm 4.06$ & $96.73 \pm 3.34$ & $96.84 \pm 3.65$ & $95.97 \pm 4.08$ & 0.743 \\
\hline MMSE & $28.64 \pm 1.43$ & $28.60 \pm 1.47$ & $28.80 \pm 1.18$ & $28.59 \pm 1.40$ & $28.80 \pm 1.26$ & 0.785 \\
\hline RAVLT: IR & $47.02 \pm 9.13$ & $48.10 \pm 11.63$ & $46.47 \pm 8.02$ & $47.30 \pm 8.76$ & $46.17 \pm 7.84$ & 0.778 \\
\hline RAVLT: STDR & $9.56 \pm 2.88$ & $9.71 \pm 3.33$ & $9.61 \pm 2.37$ & $9.48 \pm 3.15$ & $9.43 \pm 2.70$ & 0.971 \\
\hline RAVLT: LTDR & $9.14 \pm 3.35$ & $9.26 \pm 3.88$ & $9.24 \pm 2.45$ & $8.84 \pm 3.47$ & $9.29 \pm 2.92$ & 0.905 \\
\hline COWAT & $41.69 \pm 12.12$ & $42.26 \pm 13.69$ & $41.75 \pm 10.80$ & $44.57 \pm 11.13$ & $40.09 \pm 11.50$ & 0.398 \\
\hline Detection & $1.49 \pm 0.15$ & $1.51 \pm 0.12$ & $1.49 \pm 0.11$ & $1.45 \pm 0.23$ & $1.50 \pm 0.12$ & 0.317 \\
\hline One back memory & $1.31 \pm 0.18$ & $1.32 \pm 0.19$ & $1.30 \pm 0.19$ & $1.33 \pm 0.14$ & $1.33 \pm 0.17$ & 0.798 \\
\hline Groton maze learning & $68.31 \pm 28.35$ & $66.02 \pm 25.81$ & $66.55 \pm 27.85$ & $66.30 \pm 25.40$ & $65.00 \pm 17.80$ & 0.993 \\
\hline IS: visual memory & $0.05 \pm 2.02$ & $-0.39 \pm 1.87$ & $-0.04 \pm 2.02$ & $0.34 \pm 2.18$ & $0.36 \pm 1.98$ & 0.290 \\
\hline HADS: anxiety & $4.52 \pm 3.25$ & $4.14 \pm 3.43$ & $4.27 \pm 3.17$ & $3.61 \pm 2.69$ & $5.31 \pm 3.27$ & 0.124 \\
\hline HADS: depression & $2.82 \pm 2.28$ & $2.24 \pm 1.86$ & $2.84 \pm 1.90$ & $2.20 \pm 1.89$ & $3.03 \pm 2.12$ & 0.127 \\
\hline MFQ & $289.42 \pm 52.39$ & $297.55 \pm 49.41$ & $293.59 \pm 50.57$ & $290.82 \pm 52.25$ & $286.57 \pm 48.93$ & 0.807 \\
\hline SF-36: PC & $71.31 \pm 17.04$ & $75.29 \pm 16.52$ & $73.53 \pm 17.88$ & $75.42 \pm 12.86$ & $66.05 \pm 16.51$ & $0.045^{c}$ \\
\hline SF-36: MC & $76.86 \pm 15.85$ & $78.42 \pm 15.75$ & $80.88 \pm 12.05$ & $80.50 \pm 13.00$ & $73.46 \pm 16.42$ & 0.090 \\
\hline
\end{tabular}

Abbreviations: APOE, apolipoprotein E; BMI, body mass index; Camcog-R, Cambridge cognitive assessment-Revised; CCRT, Cambridge contextual reading test; COWAT, controlled oral word association test; HADS, hospital anxiety and depression scale; IPAQ, international physical activity questionnaire; IQ, intelligence quotient; IR, immediate recall; IS, index score; LTDR, long-term delayed recall; MFQ, memory functioning questionnaire; MMSE, mini-mental state examination; PG, post graduation; RAVLT, Rey auditory verbal learning test; SF36:PC, short form 36: physical component; SF36:MC, short form 36: mental component; STDR, short-term delayed recall. ${ }^{a}$ Values are expressed as mean \pm s.d. unless otherwise indicated. ${ }^{\text {b }}$ For all continuous variables, an analysis of variance was used and for all categorical variables, a chi-square test was used. Indicates the group differences significant at the 0.05 level.

(PA, $n=11 ; \mathrm{CS}, n=14$; combined, $n=10$ and control, $n=10$ ) had an FDG-PET scan at week 16 . NeuroStat three-dimensional stereotactic surface projection $z$-score images indicating regions of hypo- and hypermetabolism for the 16-week post intervention are shown in Figure 3 . The CS group showed a trend towards a metabolic increase (peak $z$-score $=4.23$ ) in the right inferior frontal gyrus, and the PA group showed a trend towards a metabolic reduction (peak $z$-score $=4.25$ ) in the left middle temporal lobe. However, the $z$-scores representing these effects did not reach statistical significance.

The volume of interest data with the results of analysis of covariance with study covariates showed significant group differences (Supplementary Table 2) for the right and left posterior cingulate cortex $(P=0.025$ and $P=0.018$, respectively) and left primary sensorimotor cortex $(P=0.045)$. Trends toward group differences were observed for left medial parietal cortex $(P=0.051)$ and left frontal lobe $(P=0.073)$. Pair-wise comparisons revealed that compared with the control group, a significant increase in metabolism was observed for the combined group $(P=0.039)$ in the left primary sensorimotor cortex (Figure 4a) with a trend towards greater activity in the left frontal lobe $(P=0.051$, Figure 4b). All other comparisons showed differences between the intervention groups but not compared with the control (data not shown) and thus did not reflect intervention-specific effects.

The findings above show moderate increases in regional counts in the left primary sensorimotor cortex in the combined group, indicating increased glucose metabolism in this region. Although it cannot be determined if this increase is from baseline, as the 


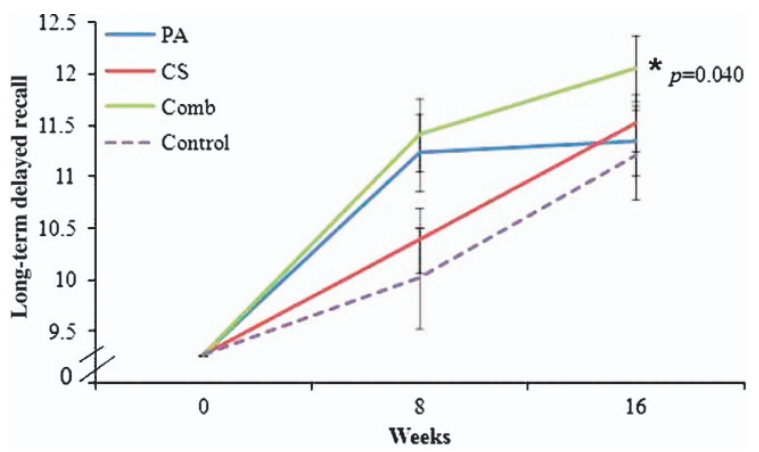

Figure 2. Effects of the interventions on long-term delayed recall (LTDR): Repeated measures analysis of covariance showed significant group differences $(P=0.040)$ for the LTDR scores. Post hoc analysis showed that only the combined group performed better when compared with the control group $(P=0.024)$. Comb, combined; CS, cognitive stimulation; PA, physical activity.

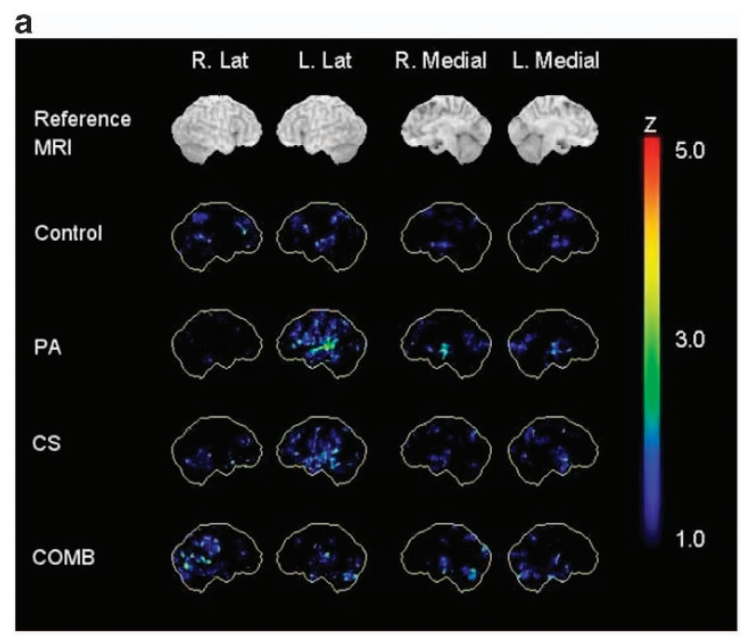

b



Figure 3. Three-dimensional stereotactic surface projection $z$-score images for the 16-week post-intervention scans showing regions of (a) hypometabolism and (b) hypermetabolism compared with baseline scans. COMB, combined; $\mathrm{CS}$, cognitive stimulation; PA, physical activity.

scans were only performed at week 16 , the data can be used to investigate associations with other outcome variables from the study. Correlation analysis was performed between regional



b



Figure 4. Effects of the intervention on regional count as determined by FDG-PET. (a) An ANCOVA showed significant group differences at 16 weeks post intervention in the left sensorimotor cortex $(P=0.045)$. Post hoc analysis showed that the combined group had more glucose uptake in this region when compared with the control group $(P=0.039)$. (b) Trends toward significant group differences $(P=0.070)$ were observed in the left frontal cortex. Post hoc analysis revealed trends toward the combined group showing increased glucose metabolism compared with the control group $(P=0.051)$. CS, cognitive stimulation; PA, physical activity.

counts in the left primary sensorimotor cortex and cognitive variables assessed at week 16 (Supplementary Table 3).

Higher regional counts within the left sensorimotor cortex and left frontal lobe correlated significantly with LTDR for the combined group only ( $P=0.030$ and $P=0.003$, respectively). Such associations were not present in any other group (Supplementary Table 3). No significant correlations were observed with baseline values for LTDR and left primary sensorimotor cortex $(\rho=0.254$, $P=0.510$ ). A positive correlation was also present between the CogState ONB (attention) task, and regional counts within the left sensorimotor cortex $(P=0.011)$ in the combined group. No correlations were observed with pre-intervention values for ONB (left primary sensorimotor cortex $(\rho=0.213, P=0.582)$ ). Unlike LTDR scores, scores for the ONB task did not improve significantly in the combined group; thus the significance of this association as it relates to improved cognition is unclear.

\section{DISCUSSION}

There are three novel findings from this study. First, this specific combination of PA and computerized brain training significantly improved verbal memory after 16 weeks. Second, this combined group showed higher regional counts compared with the control group; indicating higher levels of glucose metabolism. Specifically, significant increases were observed in the left primary sensorimotor cortex. Third, higher regional counts were associated with 
improved verbal memory at week 16 post intervention in the combined group only. This association was not present in any other group, indicating that higher neuronal activity could be due to the activities being combined.

Consistent with our current findings, previous studies have reported that a combination of physical and mental activities are more beneficial. ${ }^{35,36}$ However, these studies included paper and pencil based training and did not include additional assessments of biomarkers. Other studies reporting improved cognition have applied stand-alone interventions of $\mathrm{PA}^{18,63}$ or $\mathrm{CS}^{31,32}$ A 6-month randomized trial of aerobic, strength, flexibility, balance and coordination exercises, or brain training in the form of operating common features of computers ( $n=256$ women, 70-93 years of age), resulted in improved immediate and delayed recall in the intervention groups. ${ }^{64}$ However, this study lacked a combined group. Our study shows that a combination of these activities, which included low intensity walking, provided benefits particularly for verbal episodic memory compared with the control group. In this context, it is noteworthy that episodic memory impairment is the core deficit of AD. ${ }^{65}$

Our study did not demonstrate post-intervention benefits for executive functions, visual memory, processing speed or attention. Limited sample size, the counterbalanced-design of our CS intervention or differences in the outcome measures may be responsible, as relationships with these particular cognitive domains have been found in other trials. ${ }^{22,31,32}$ It may also suggest that the intensity of PA may be more important than the type of exercise for these specific cognitive domains. For example, aerobic exercise has been linked specifically with improved attention $^{66}$ and executive control processes. ${ }^{14,67}$ Given the age of the participants, the PA intervention in the current study was deliberately kept mild and hence was not aerobic in nature. A previous study showed that aerobic exercise in the form of brisk walking generated positive outcomes, but only if practiced for at least 1 year. ${ }^{68} \mathrm{~A}$ recent trial using PA and identical brain training programs for 12 weeks reported improved global cognitive scores over time, but these scores did not differ between the intervention and the active control group. ${ }^{34}$ Our results demonstrate the cognitive benefits of combined activities after 16 weeks of training.

Unexpectedly, the control group reported better memory performance at 16 weeks post intervention on the MFQ in this study. Memory self-appraisal and depression tendencies are strongly associated. ${ }^{69}$ It is possible that participating in such a trial may have some placebo-like effect; that is, encouraging an imitation of treatment and resulting in a self-reported improvement in memory or a decrease in subjective memory complaints. However, this finding was not supported by any objective measure of cognitive function included in the current study that could possibly explain the improved memory self-perception, nor did the control subjects demonstrate any changes in their physical fitness assessments post intervention.

Our study suggests that the observed benefits in verbal memory may be mediated by the higher glucose metabolism levels in the left sensorimotor cortex. To the best of our knowledge, there is no other study that has investigated the benefits of single as well as combined lifestyle interventions using FDG-PET. FDG-PET has been used in one study to examine a cohort of 17 healthy older adults, to investigate the benefits of a combination of memory training, PA, stress reduction and diet. The study reported a $5 \%$ decrease in activity in the left dorsolateral prefrontal cortex in the intervention group. ${ }^{70}$ The intervention used in the study by Small et al. ${ }^{70}$ was a combination aimed at providing an overall healthier lifestyle, however, it is not possible to interpret whether all or any one component of this healthy lifestyle caused the resulting improvement in word fluency and the observed change in brain activity.
In addition, we showed that of all the variables, only the LTDR was positively associated with the observed higher regional count. This suggests that the increased LTDR scores could be due to the higher neuronal activity in this brain region. The left primary sensorimotor cortex is usually associated with motor functioning, although animal studies suggest that it is highly dynamic. Thus, cognitive functioning could be mediated in this area because it shows high neuronal plasticity, and increased activation within this area is seen following long-term learning. ${ }^{71,72}$ Unlike the frontal cortex, this brain region is relatively spared from $A D$ pathology and does not show reductions in glucose metabolism in early AD. ${ }^{44}$ According to the cognitive reserve hypothesis, there is increased and decreased activation in specific brain regions during task performance reflecting the complexity of the neural expression of the cognitive reserve. It has been speculated that for a given task, high cognitive reserve could result in reduced cerebral activation in regions normally specifically engaged to enable performance of the task, and this could possibly explain why we found an increase in the left primary sensorimotor cortex whereas Small et al. ${ }^{70}$ had found a decrease in the dorsolateral prefrontal cortex. It is tempting to speculate that, as the more susceptible areas of the brain are affected in early dementia, the more resistant areas such as the sensorimotor cortex may provide cognitive reserve and higher neuronal activity following lifestyle interventions. $^{73}$

There are some limitations to this study. The participants were not completely randomized. Although randomization is important, such an approach may not be practical in lifestyle intervention trials. Cognitively healthy individuals are often highly motivated, usually active and request to be placed into groups that are rewarding. Internet publicity of brain training games, social interaction and spouses/friends of study participants requesting to be placed in the same group make it more challenging to ensure randomization. However, baseline values were taken as a covariate in the analysis to reduce any nonrandomization bias. Another limitation is that the FDG-PET scans were performed at week 16 post intervention only. Thus it cannot be determined if the reported increase is from baseline.

In conclusion, this study adds to the clinical significance of lifestyle interventions by demonstrating improved cognition following 16 weeks of walking and resistance training together with computerized visual and auditory cognitive training. The results identify higher cerebral glucose metabolism as a result of these activities, beyond the traditional benefits of enhanced blood circulation as observed for both physical and mental activities. Although data from the FDG-PET scans must be interpreted with caution, the current findings provide information that combined lifestyle activities may be altering neuronal activity. A larger sample size with longitudinal follow-up would consolidate findings and provide further information. Specifically, it would help to determine if such changes in cerebral glucose metabolism are maintained following completion of the intervention, or if the activities need to be continued to maintain these benefits. The findings do indicate that the type of activities trialed here provide greater cognitive benefits than routine lifestyle activities in the healthy elderly.

\section{CONFLICT OF INTEREST}

The authors declare no conflict of interest.

\section{ACKNOWLEDGMENTS}

TS is supported by the Australian Postgraduate Award from the University of Western Australia, the Research Excellence Award form Edith Cowan University and the Freemasons of Western Australia Education Grant 2010 and 2011. The McCusker Alzheimer's Research Foundation contributed financial and in-kind support for the study. Posit Science (San Francisco, CA, USA) has generously provided us their 
software programs for the study. We thank all the study participants for their time, commitment and dedication for this intervention study and also the research staff at the Foundation.

\section{REFERENCES}

1 World Health Organization. Dementia: a Public Health Priority. World Health Organization: Geneva, Switzerland, 2012.

2 de Bruijn RF, Schrijvers EM, de Groot KA, Witteman JC, Hofman A, Franco OH et al. The association between physical activity and dementia in an elderly population: the Rotterdam Study. Eur J Epidemiol. 2013; 28: 277-283.

3 Barberger-Gateau P, Raffaitin C, Letenneur L, Berr C, Tzourio C, Dartigues J-F et al. Dietary patterns and risk of dementia The Three-City cohort study. Neurology 2007; 69: 1921-1930.

4 Scherder EJ, Bogen T, Eggermont LH, Hamers JP, Swaab DF. The more physical inactivity, the more agitation in dementia. Int Psychogeriatr 2010; 22: 1203-1208.

5 Access Economics. Keeping dementia front of mind: incidence and prevalence 2009-2050. Alzheimer's Australia, 2009. Available at http://www.alzheimers.org au/upload/Front_of_Mind_Full_Rep ort1 pdf.

6 Mattson MP, Duan W, Chan SL, Cheng A, Haughey N, Gary DS et al. Neuroprotective and neurorestorative signal transduction mechanisms in brain aging: modification by genes, diet and behavior. Neurobiol Aging 2002; 23: 695-706.

7 Studenski S, Carlson MC, Fillit H, Greenough WT, Kramer A, Rebok GW. From bedside to bench: does mental and physical activity promote cognitive vitality in late life? Sci Aging Knowl Environ 2006; 2006: pe21.

8 Fillit HM, Butler RN, O'Connell AW, Albert MS, Birren JE, Cotman CW et al. Achieving and maintaining cognitive vitality with aging. Mayo Clin Proc 2002; 77: 681-696.

9 Abbott RD, White LR, Ross GW, Masaki KH, Curb JD, Petrovitch H. Walking and dementia in physically capable elderly men. JAMA 2004; 292: 1447-1453.

10 Weuve J, Kang JH, Manson JAE, Breteler M, Ware JH, Grodstein F. Physical activity, including walking, and cognitive function in older women. JAMA 2004; 292: 1454.

11 Yaffe K, Barnes D, Nevitt M, Lui LY, Covinsky K. A prospective study of physical activity and cognitive decline in elderly women: women who walk. Arch Intern Med 2001; 161: 1703.

12 Laurin D, Verreault R, Lindsay J, MacPherson K, Rockwood K. Physical activity and risk of cognitive impairment and dementia in elderly persons. Arch Neurol 2001; 58: 498-504.

13 Blumenthal JA, Madden DJ. Effects of aerobic exercise training, age, and physical fitness on memory-search performance. Psychol Aging 1988; 3: 280-285.

14 Colcombe S, Kramer AF. Fitness effects on the cognitive function of older adults: a meta-analytic study. Psychol Sci 2003; 14: 125-130.

15 Renaud M, Maquestiaux F, Joncas S, Kergoat M-J, Bherer L. The effect of three months of aerobic training on response preparation in older adults. Front Aging Neurosci 2010; 2: 148.

16 Palleschi L, Vetta F, De Gennaro E, Idone G, Sottosanti G, Gianni W et al. Effect of aerobic training on the cognitive performance of elderly patients with senile dementia of Alzheimer type. Arch Gerontol Geriatr 1996; 22: 47-50.

17 Rolland Y, Rival L, Pillard F, Lafont C, Riviere D, Albarede JL et al. Feasibily of regular physical exercice for patients with moderate to severe alzheimer disease. $J$ Nutr Health Aging 2000; 4: 109-113.

18 Lautenschlager NT, Cox KL, Flicker L, Foster JK, van Bockxmeer FM, Xiao J et al. Effect of physical activity on cognitive function in older adults at risk for Alzheimer disease: a randomized trial. JAMA 2008; 300: 1027-1037.

19 Erickson KI, Voss MW, Prakash RS, Basak C, Szabo A, Chaddock L et al. Exercise training increases size of hippocampus and improves memory. Proc Natl Acad SCi USA 2011; 108: 3017

20 Lachman ME, Neupert SD, Bertrand R, Jette AM. The effects of strength training on memory in older adults. J Aging Phys Act 2006; 14: 59.

21 Cassilhas RC, Viana VAR, Grassmann V, Santos RT, Santos RF, Tufik S et al. The impact of resistance exercise on the cognitive function of the elderly. Med Sci Sports Exerc 2007; 39: 1401-1407.

22 Ball K, Berch DB, Helmers KF, Jobe JB, Leveck MD, Marsiske M et al. Effects of cognitive training interventions with older adults: a randomized controlled trial. JAMA 2002; 288: 2271-2281.

23 Calero MD, Navarro E. Cognitive plasticity as a modulating variable on the effects of memory training in elderly persons. Arch Clin Neuropsychol 2007; 22: 63-72.

24 Engvig A, Fjell AM, Westlye LT, Moberget T, Sundseth $\varnothing$, Larsen VA et al. Effects of memory training on cortical thickness in the elderly. Neuroimage 2010; 52: 1667-1676.

25 Rozzini L, Costardi D, Chilovi BV, Franzoni S, Trabucchi M, Padovani A. Efficacy of cognitive rehabilitation in patients with mild cognitive impairment treated with cholinesterase inhibitors. Int J Geriatr Psychiatry 2007; 22: 356-360.
26 Farina E, Fioravanti R, Chiavari L, Imbornone E, Alberoni M, Pomati S et al. Comparing two programs of cognitive training in Alzheimer's disease: a pilot study. Acta Neurol Scand 2002; 105: 365-371.

27 Zanetti O, Binetti G, Magni E, Rozzini L, Bianchetti A, Trabucchi M. Procedural memory stimulation in Alzheimer's disease: impact of a training programme. Acta Neurol Scand 2009; 95: 152-157.

28 Zanetti O, Zanieri G, Di Giovanni G, De Vreese LP, Pezzini A, Metitieri T et al. Effectiveness of procedural memory stimulation in mild Alzheimer's disease patients: a controlled study. Neuropsychol Rehabil 2001; 11: 263-272.

29 Wilson RS, Bennett DA, Bienias JL, Aggarwal NT, Mendes de Leon CF, Morris MC et al. Cognitive activity and incident AD in a population-based sample of older persons. Neurology 2002; 59: 1910-1914.

30 Wilson RS, Scherr PA, Schneider JA, Tang Y, Bennett DA. Relation of cognitive activity to risk of developing Alzheimer disease. Neurology 2007; 69: 1911-1920.

31 Mahncke HW, Connor BB, Appelman J, Ahsanuddin ON, Hardy JL, Wood RA et al. Memory enhancement in healthy older adults using a brain plasticity-based training program: a randomized, controlled study. Proc Natl Acad Sci USA 2006; 103: $12523-12528$.

32 Smith GE, Housen P, Yaffe K, Ruff R, Kennison RF, Mahncke HW et al. A cognitive training program based on principles of brain plasticity: results from the Improvement in Memory with Plasticity-based Adaptive Cognitive Training (IMPACT) Study. J Am Geriatr Soc 2009; 57: 594-603.

33 Anguera J, Boccanfuso J, Rintoul J, Al-Hashimi O, Faraji F, Janowich J et al. Video game training enhances cognitive control in older adults. Nature 2013; 501: 97-101.

34 Barnes DE, Santos-Modesitt W, Poelke Gina, Kramer Arthur F, Castro Cynthia, Middleton Laura E et al. The mental activity and exercise (max) trial: a randomized controlled trial to enhance cognitive function in older adults. JAMA Intern Med 2013; 173: 797-804.

35 Fabre C, Chamari K, Mucci P, Masse-Biron J, Prefaut C. Improvement of cognitive function by mental and/or individualized aerobic training in healthy elderly subjects. Int J Sports Med 2002; 23: 415-421.

36 Oswald WD, Gunzelmann T, Rupprecht R, Hagen B. Differential effects of single versus combined cognitive and physical training with older adults: the SimA study in a 5-year perspective. Eur J Ageing 2006; 3: 179-192.

37 Shatil E. Does combined cognitive training and physical activity training enhance cognitive abilities more than either alone? A four-condition randomized controlled trial among healthy older adults. Front Aging Neurosci 2013; 5: 8.

38 Sokoloff L. Localization of functional activity in the central nervous system by measurement of glucose utilization with radioactive deoxyglucose. J Cereb Blood Flow Metab 1981; 1: 7-36.

39 Frackowiak RS Magistretti PJ Shulman RG Altman JS Adams M. Neuroenergetics: Relevance for Functional Brain Imaging. Human Frontier Science Program: Strasbourg, France, 2001

40 Drzezga A, Lautenschlager N, Siebner $H$, Riemenschneider M, Willoch F, Minoshima $\mathrm{S}$ et al. Cerebral metabolic changes accompanying conversion of mild cognitive impairment into Alzheimer's disease: a PET follow-up study. Eur J NuCl Med Mol Imag 2003; 30: 1104-1113.

41 Nestor PJ, Fryer TD, Smielewski P, Hodges JR. Limbic hypometabolism in Alz heimer's disease and mild cognitive impairment. Ann Neurol 2003; 54: 343-351.

42 Minoshima S, Frey KA, Koeppe RA, Foster NL, Kuhl DE. A diagnostic approach in Alzheimer's Disease using three-dimensional stereotactic surface. J Nucl Med 1995; 36: 1238-1248.

43 Masdeu JC, Zubieta JL, Arbizu J. Neuroimaging as a marker of the onset and progression of Alzheimer's disease. J Neurol Sci 2005; 236: 55-64.

44 Minoshima S, Giordani B, Berent S, Frey KA, Foster NL, Kuhl DE. Metabolic reduction in the posterior cingulate cortex in very early Alzheimer's disease. Ann Neurol 1997; 42: 85-94.

45 Hoffman JM, Welsh-Bohmer KA, Hanson M, Crain B, Hulette C, Earl N et al. FDG PET imaging in patients with pathologically verified dementia. J Nucl Med 2000; 41: 1920-1928.

46 Förster S, Buschert VC, Buchholz HG, Teipel SJ, Friese U, Zach C et al. Effects of a 6month cognitive intervention program on brain metabolism in amnestic mild cognitive impairment and mild Alzheimer's disease. J Alzheimers Dis 2011; 25: 695-706.

47 Tashiro $\mathrm{M}$, Itoh $\mathrm{M}$, Fujimoto $\mathrm{T}$, Fujiwara $\mathrm{T}$, Ota $\mathrm{H}$, Kubota $\mathrm{K}$ et al. 18F-FDG PET mapping of regional brain activity in runners. J Sports Med Phys Fitness 2001; 41 $11-17$.

48 Kemppainen J, Aalto S, Fujimoto T, Kalliokoski KK, Långsjö J, Oikonen V et al. High intensity exercise decreases global brain glucose uptake in humans. J Physiol 2005; 568: 323-332.

49 Borg G. Borg's Perceived Exertion and Pain Scales. Human Kinetics Publishers: Champaign, IL, US, 1998.

50 Wenham PR, Newton CR, Price WH. Analysis of apolipoprotein E genotypes by the Amplification Refractory Mutation System. Clin Chem 1991; 37: 241-244. 
51 Beardsall L, Huppert FA. Improvement in NART word reading in demented and normal older persons using the Cambridge Contextual Reading Test. J Clin Exp Neuropsychol 1994; 16: 232-242.

52 Rey A. L'examen clinique en psychologie [Clinical tests in psychology]. Presses Universitaires de France: Paris, France, 1964.

53 Benton AL, Hamsher KS. Multilingual Aphasia Examination (Manual, revised, 1978). University of lowa: lowa City, IA, USA, 1976.

54 Roth M, Tym E, Mountjoy CQ, Huppert FA, Hendrie H, Verma S et al. CAMDEX. A standardised instrument for the diagnosis of mental disorder in the elderly with special reference to the early detection of dementia. Br J Psychiatry 1986; 149: 698-709.

55 Zigmond AS, Snaith RP. The hospital anxiety and depression scale. Acta Psychiatr Scand 1983; 67: 361-370.

56 Gilewski MJ, Zelinski EM, Schaie KW. The Memory Functioning Questionnaire for assessment of memory complaints in adulthood and old age. Psychol Aging 1990; 5: 482-490.

57 Ware JE Jr, Gandek B. Overview of the SF-36 Health Survey and the International Quality of Life Assessment (IQOLA) Project. J Clin Epidemiol 1998; 51: 903-912.

58 Singh SJ, Morgan MD, Scott S, Walters D, Hardman AE. Development of a shuttle walking test of disability in patients with chronic airways obstruction. Thorax 1992; 47: 1019-1024.

59 Minoshima S, Koeppe RA, Frey KA, Ishihara M, Kuhl DE. Stereotactic PET atlas of the human brain: aid for visual interpretation of functional brain images. J Nucl Med 1994; 35: 949-954.

60 Minoshima S, Koeppe RA, Frey KA, Kuhl DE. Anatomic standardization: linear scaling and nonlinear warping of functional brain images. J Nucl Med 1994; 35: 1528-1537.

61 Perneger TV. What's wrong with Bonferroni adjustments. BMJ 1998; 316: 1236-1238. deoxy-D-glucose (FDG)-PET in APOE\&4 carriers in the Australian population. $J$ Alzheimers Dis 2008; 13: 137-146.

63 Ruscheweyh R, Willemer C, Krüger K, Duning T, Warnecke T, Sommer J et al. Physical activity and memory functions: an interventional study. Neurobiol Aging 2011; 32: 1304-1319.

64 Klusmann V, Evers A, Schwarzer R, Schlattmann P, Reischies FM, Heuser I et al. Complex mental and physical activity in older women and cognitive performance:
62 Rimajova M, Lenzo NP, Wu J-S, Bates KA, Campbell A, Dhaliwal SS et al. Fluoro-2-

a 6-month randomized controlled trial. J Gerontol A Biol Sci Med Sci 2010; 65: 680-688.

65 Aggarwal NT, Wilson RS, Beck TL, Bienias JL, Bennett DA. Mild cognitive impairment in different functional domains and incident Alzheimer's disease. J Neurol Neurosurg Psychiatry 2005; 76: 1479-1484.

66 Colcombe SJ, Kramer AF, Erickson KI, Scalf P, McAuley E, Cohen NJ et al. Cardiovascular fitness, cortical plasticity, and aging. Proc Natl Acad Sci USA. 2004; 101: 3316-3321.

67 Kramer AF, Hahn S, Cohen NJ, Banich MT, McAuley E, Harrison CR et al. Ageing, fitness and neurocognitive function. Nature 1999; 400: 418-419.

68 Voss MW, Prakash RS, Erickson KI, Basak C, Chaddock L, Kim JS et al. Plasticity of brain networks in a randomized intervention trial of exercise training in older adults. Front Aging Neurosci 2010; 2: pii:32.

69 Antikainen R, Hänninen T, Honkalampi K, Hintikka J, Koivumaa-Honkanen H, Tanskanen A et al. Mood improvement reduces memory complaints in depressed patients. Eur Arch Psychiatry Clin Neurosci 2001; 251: 6-11.

70 Small GW, Silverman DHS, Siddarth P, Ercoli LM, Miller KJ, Lavretsky H et al. Effects of a 14-day healthy longevity lifestyle program on cognition and brain function. Am J Geriatr Psychiatry 2006; 14: 538-545.

71 Matsuzaka Y, Picard N, Strick PL. Skill representation in the primary motor cortex after long-term practice. J Neurophysiol 2007; 97: 1819-1832.

72 Rokni U, Richardson AG, Bizzi E, Seung HS. Motor learning with unstable neural representations. Neuron 2007; 54: 653-666.

73 Salustri C, Tecchio F, Zappasodi F, Tomasevic L, Ercolani M, Moffa F et al. Sensorimotor cortex reorganization in alzheimer's disease and metal dysfunction: a MEG Study. Int J Alzheimers Dis 2013; 2013: 638312.

(c) $($ ) $\$$ This work is licensed under a Creative Commons Attribution-

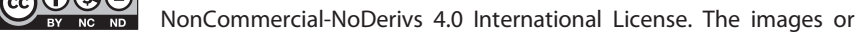
other third party material in this article are included in the article's Creative Commons license, unless indicated otherwise in the credit line; if the material is not included under the Creative Commons license, users will need to obtain permission from the license holder to reproduce the material. To view a copy of this license, visit http:// creativecommons.org/licenses/by-nc-nd/4.0/

Supplementary Information accompanies the paper on the Translational Psychiatry website (http://www.nature.com/tp) 